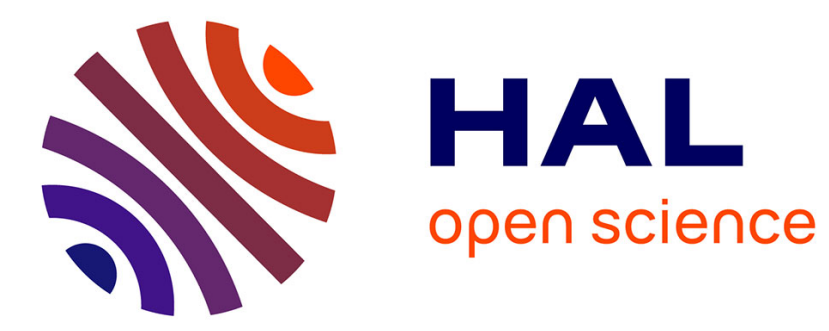

\title{
Observer-based relay feedback controller design for LTI systems
}

Zohra Kader, Christophe Fiter, Laurentiu Hetel, Lotfi Belkoura

\section{To cite this version:}

Zohra Kader, Christophe Fiter, Laurentiu Hetel, Lotfi Belkoura. Observer-based relay feedback controller design for LTI systems. ECC 2016 - European Control Conference, Jun 2016, Aalborg, Denmark. pp.1667-1672, 10.1109/ECC.2016.7810530 . hal-01415095

\section{HAL Id: hal-01415095 \\ https://hal.science/hal-01415095}

Submitted on 23 Jan 2018

HAL is a multi-disciplinary open access archive for the deposit and dissemination of scientific research documents, whether they are published or not. The documents may come from teaching and research institutions in France or abroad, or from public or private research centers.
L'archive ouverte pluridisciplinaire HAL, est destinée au dépôt et à la diffusion de documents scientifiques de niveau recherche, publiés ou non, émanant des établissements d'enseignement et de recherche français ou étrangers, des laboratoires publics ou privés. 


\title{
Observer-based relay feedback controller design for LTI systems
}

\author{
Zohra Kader, Christophe Fiter, Laurentiu Hetel and Lotfi Belkoura .
}

\begin{abstract}
This paper presents a design approach for observer-based relay feedback controllers. A switching law dependent on the estimation state is designed while using a Luenberger observer. The stabilization problem leads to qualitative conditions. A numerical example is provided to assess the effectiveness of the developed method.
\end{abstract}

\section{INTRODUCTION}

Relays are largely studied in control theory since the fifties [8], [27]. They are widely used in different application fields and for different targets - see for instance [13], [18], [29], [30]. Relay feedback controllers present some advantages which make them a perfect substitute to continuous control laws (see for instance [11], [14], [27], [28]). Indeed, they can emulate locally the behavior of a linear static feedback, and they are classified as simple and robust controller [7], [14], [23]. However, the usefulness of relays for stabilization and control does not exclude difficulties and some undesired phenomena. From a theoretical point of view, systems with relay feedback control can be seen as switched systems [17] with a complex behaviour. The design of a relay feedback controller is not an obvious problem even for the case of linear systems. In the literature [15], [16], the presence of sliding modes, limit cycles and chattering in relay feedback systems is pointed out. These phenomena must not be neglected and their study is theoretically challenging. In particular, for systems with sliding modes the notion of system's solution must be reviewed to take into account the dynamics obtained by fast switching [5], [9]. Frequency domain methods [3] and LMI approaches [22], [23] have also been used for relay feedback controller design. Recently, a convex embedding formalism has been used in order to design relay feedback controllers in [14] and [12]. However, to the best of our knowledge, the existing results about relay feedback systems consider the system's states as perfectly known. In many practical cases, the state of the system is not

\footnotetext{
*This work was supported by the Agence Nationale pour la Recherche Project ROCC-SYS under the Grant ANR-14-C27-0008.

Zohra Kader is with CRIStAL CNRS UMR 9189, Université Lille 1 , Sciences et Technologies, Villeneuve d'Ascq, France. She is also with Non-A, INRIA - Lille Nord Europe, Villeneuve d'Ascq, France. zohra.kader@inria.fr.

Christophe Fiter is with CRIStAL CNRS UMR 9189, Université Lille 1, Sciences et Technologies, Villeneuve d'Ascq, France Christophe.Fiter@univ-lillel.fr.

Laurentiu Hetel is with CRIStAL CNRS UMR 9189, École Centrale de Lille, Villeneuve d'Ascq, France laurentiu.heteleec-lille.fr.

Lotfi Belkoura is with CRIStAL CNRS UMR 9189, Université Lille 1, Sciences et Technologies, Villeneuve d'Ascq, France. He is also with Non-A, INRIA - Lille Nord Europe, Villeneuve d'Ascq, France Lotfi.Belkouraduniv-lillel.fr
}

fully available for measurements. In this case an observerbased controller must be designed.

Here, we design a relay feedback controller with an observer-based switching law. Using a convex embedding formalism [6], [14], it will be shown how we can design an observer and switching hyperplane so as to ensure the local exponential stability of the closed loop system. The research here is also related with the output feedback sliding mode control problem [21], [10] and output feedback design for switched systems [25].

The paper is organized as follows: Section II gives the system description and exposes the problem under study. A qualitative stability result is proposed in Section III. In Section IV, a numerical example is given to illustrate the efficiency of the presented method. Finally, perspectives are given in the last section together with the conclusion.

\section{A. Notations}

In this paper we use the notation $\mathbb{R}^{+}$to refer to the interval $[0, \infty)$. The transpose of a matrix $M$ is denoted by $M^{T}$ and if the matrix is symmetric the symmetric elements are denoted by $*$. The notation $M \succeq 0$ (resp. $M \preceq 0$ ) means that the matrix $M$ is positive (resp. negative) semidefinite, and the notation $M \succ 0$ (resp. $M \prec 0$ ) means that it is positive definite (resp. negative definite). The identity matrix is denoted by $I$ and both notations $\operatorname{eig}_{\min }(M)$ and $\operatorname{eig}_{\max }(M)$ are used to refer to the minimum and maximum eigenvalue respectively of a matrix $M$. For a positive definite matrix $P \in \mathbb{R}^{n \times n}$ and a positive scalar $\gamma$, we denote by $\mathcal{E}(P, \gamma)$ the ellipsoid

$$
\mathcal{E}(P, \gamma)=\left\{x \in \mathbb{R}^{n}: x^{T} P x \leq \gamma\right\},
$$

and for all positive scalar $r$.

For a given set $\mathcal{S}$, the notation $\operatorname{Conv}\{\mathcal{S}\}$ indicates the convex hull of the set, $\operatorname{int}\{\mathcal{S}\}$ its interior and $\overline{\mathcal{S}}$ its closure and finally the closed convex hull of the set $\mathcal{S}$ will be noted by $\overline{\operatorname{Conv}}\{\mathcal{S}\}$. The minimum argument of a given function $f: \mathcal{S} \longrightarrow \mathbb{R}$ such that the set $\mathcal{S} \subset \mathbb{R}$ is a finite set of vectors is noted by

$$
\arg \min f=\{y \in \mathcal{S}: f(y) \leq f(z), \forall z \in \mathcal{S}\} .
$$

For a positive integer $N$, we denote by $\mathcal{I}_{N}$ the set $\{1, \ldots, N\}$. By $\Delta_{N}$ we denote the unit simplex

$$
\Delta_{N}=\left\{\alpha=\left[\begin{array}{c}
\alpha_{1} \\
\vdots \\
\alpha_{N}
\end{array}\right] \in \mathbb{R}^{N}: \sum_{i=1}^{N} \alpha_{i}=1, \alpha_{i} \geq 0, i \in \mathcal{I}_{N}\right\} .
$$




\section{SySTEM DESCRIPTION AND PROBLEM STATEMENT}

\section{A. System description}

Consider the linear system

$$
\left\{\begin{array}{l}
\dot{x}=A x+B u, \\
y=C x,
\end{array}\right.
$$

with $x \in \mathbb{R}^{n}$, an input $u$ which takes values in the set $\mathcal{V}=$ $\left\{v_{1}, \ldots, v_{N}\right\} \subset \mathbb{R}^{m}$ and an output $y \in \mathbb{R}^{p} . A \in \mathbb{R}^{n \times n}$, $B \in \mathbb{R}^{n \times m}$ and $C \in \mathbb{R}^{p \times n}$ are the matrices describing the system.

In the sequel we assume that:

A-1 The pair $(A, B)$ is stabilizable, which means that there exists a matrix $K$ such that the closed-loop matrix $A_{c l}=A+B K$ is Hurwitz.

A-2 The set $\operatorname{int}\{\operatorname{Conv}\{\mathcal{V}\}\}$ is nonempty and the null vector is contained inside $(0 \in \operatorname{int}\{\operatorname{Conv}\{\mathcal{V}\}\})$.

A-3 The pair $(A, C)$ is detectable, which means that there exists a matrix $L$ such that the matrix $A_{o}=A+L C$ is Hurwitz.

This paper deals with the stabilization of system (4) in the case of an observer-based switching law. We consider a controller given by

$$
u(\hat{x}) \in \underset{v \in \mathcal{V}}{\arg \min } \hat{x}^{T} \Gamma v,
$$

where the matrix $\Gamma \in \mathbb{R}^{n \times m}$ characterizes the switching hyperplanes, and $\hat{x} \in \mathbb{R}^{n}$ is the estimated state which is computed by the full-order Luenberger state observer [19], [20]

$$
\left\{\begin{array}{l}
\dot{\hat{x}}=A \hat{x}+B u+L(\hat{y}-y), \\
\hat{y}=C \hat{x} .
\end{array}\right.
$$

The formulation of the controller (5) encompasses the classical sign function in the classical relay feedback. Note that, if $\mathcal{V}=\left\{v_{1}, v_{2}\right\}=\{-v, v\}$ with $v>0$ then, we get

$$
\begin{aligned}
u(\hat{x}) & =-v \operatorname{sign}(\Gamma \hat{x}) \\
& \in\left\{\begin{array}{l}
v \text { if } \Gamma \hat{x}<0, \\
\{-v, v\} \text { if } \Gamma \hat{x}=0, \\
v \text { if } \Gamma \hat{x}>0 .
\end{array}\right.
\end{aligned}
$$

Our objective is to provide conditions which guarantee the existence of matrices $\Gamma$ (which characterizes the switching hyperplanes of the control law) and $L$ (the observer gain) such that the closed-loop system

$$
\left[\begin{array}{c}
\dot{x} \\
\dot{\hat{x}}
\end{array}\right]=\left[\begin{array}{cc}
A & 0 \\
L C & A+L C
\end{array}\right]\left[\begin{array}{l}
x \\
\hat{x}
\end{array}\right]+\left[\begin{array}{l}
B \\
B
\end{array}\right] u
$$

with the control law (5) is locally exponentially stable (this problem will be mathematically formalized farther in II-C).

\section{B. Solution concept}

Using the augmented state

$$
\xi=\left[\begin{array}{l}
\hat{x} \\
e
\end{array}\right]=\left[\begin{array}{cc}
0 & I \\
-I & I
\end{array}\right]\left[\begin{array}{l}
x \\
\hat{x}
\end{array}\right],
$$

where $e=\hat{x}-x$ is the estimation error, the interconnection (4), (6) can be written as the augmented closed-loop system

$$
\left\{\begin{aligned}
\dot{\xi} & =\left[\begin{array}{cc}
A & L C \\
0 & A+L C
\end{array}\right] \xi+\left[\begin{array}{c}
B \\
0
\end{array}\right] u(\hat{x}), \\
y & =\left[\begin{array}{ll}
C & C
\end{array}\right] \xi,
\end{aligned}\right.
$$

which leads to

$$
\left\{\begin{array}{l}
\dot{\xi}=\tilde{A} \xi+\tilde{B} \bar{u}(\xi)=\mathcal{X}(\xi), \\
y=\left[\begin{array}{ll}
C & C
\end{array}\right] \xi,
\end{array}\right.
$$

$$
\begin{aligned}
& \text { where } \tilde{A}=\left[\begin{array}{cc}
A & L C \\
0 & A+L C
\end{array}\right], \tilde{B}=\left[\begin{array}{c}
B \\
0
\end{array}\right] \text {, and } \\
& \bar{u}(\xi)=u\left(\left[\begin{array}{ll}
I & 0
\end{array}\right] \xi\right)=u(\hat{x}) \in \underset{v \in \mathcal{V}}{\arg \min } \xi^{T}\left[\begin{array}{l}
I \\
0
\end{array}\right] \Gamma v .
\end{aligned}
$$

Note that this is a differential equation with a discontinuous right hand side [9], [5], and thus we need an appropriate formalism and specific tools to define the system's solutions and analyze their behaviour.

Therefore, to the discontinuous closed-loop system (11), (12) we associate the differential inclusion

$$
\dot{\xi} \in \mathcal{F}[\mathcal{X}](\xi),
$$

with $\mathcal{F}[\mathcal{X}](\xi)$ the set-valued map which can be computed from the differential equation with a discontinuous right hand side using the construction given in [2], [5], [9], [24]

$$
\mathcal{F}[\mathcal{X}](\xi)=\bigcap_{\delta>0} \bigcap_{\mu(\mathcal{S})=0} \overline{\operatorname{Conv}}\{\mathcal{X}(\mathcal{B}(\xi, \delta)) \backslash \mathcal{S}\}, \xi \in \mathbb{R}^{2 n},
$$

where $\overline{\mathrm{Conv}}$ is the closed convex hull, $\mathcal{B}(\xi, \delta)$ is the open ball centered on $\xi$ with radius $\delta$, and $\mathcal{S}$ is a set of measure zero with $\mu(\mathcal{S})$ its measure in the sense of Lebesgue. The closedloop system is then modeled by a differential inclusion for which the notion of a solution was defined in [9], and recalled hereafter.

Definition 1: (Filippov solution) Consider the closed-loop system (11) and its associated differential inclusion (13). A Filippov solution of the discontinuous system (11), (12) over the interval $\left[t_{a}, t_{b}\right] \subset[0, \infty)$ is an absolutely continuous mapping $y(t):\left[t_{a}, t_{b}\right] \longrightarrow \mathbb{R}^{2 n}$ satisfying:

$$
\dot{y}(t) \in \mathcal{F}[\mathcal{X}](y(t)), \quad \text { for almost all } t \in\left[t_{a}, t_{b}\right],
$$

with $\mathcal{F}[\mathcal{X}](\xi)$ given by (14).

A differential inclusion has at least one solution if the set valued map $\mathcal{F}[\mathcal{X}](\xi)$ is locally bounded and takes nonempty, compact and convex values [1], [2], [5], [9] which is the case of the differential inclusion (13) corresponding to the system (11), (12).

\section{Problem statement}

Hereafter the notion of stability which will be used is introduced and we mathematically formalize the problem under study.

Definition 2: (local exponential stability) The differential inclusion (13) is said to be locally exponentially stable with a decay rate $\alpha$ (it is also said to be locally $\alpha$-stable) to the origin in a compact set $\Omega$ containing the origin if there exist 
positive scalars $c$ and $\alpha$ such that every possible solution $\xi(t)$ of (13) starting from any initial condition $\xi(0) \in \Omega$ verifies

$$
\|\xi(t)\| \leq c e^{-\alpha t}\|\xi(0)\| .
$$

We recall that sufficient conditions for the local exponential stability with decay rate $\alpha$ of a differential equation with a discontinuous right hand side $\dot{\xi}=\mathcal{X}(\xi)$, with $\mathcal{X}$ locally bounded, are given by the existence of a strict Lyapunov function $V, V(0)=0, V(\xi)>0, \forall \xi \neq 0$, such that

$$
\sup _{y \in \mathcal{F}[\mathcal{X}](\xi)} \frac{\partial V}{\partial \xi} y \leq-2 \alpha V(\xi), \forall \xi \in \mathcal{D}
$$

for some positive scalar $\alpha$ and a domain $\mathcal{D}$ such that $0 \in$ $\operatorname{int}\{\mathcal{D}\}$.

The main problem under study is mathematically formulated as follows:

Problem. Are there matrices $\Gamma$ and $L$ such that system (11)-(12) is locally exponentially stable (when solutions are understood in the sense of Filippov)?

\section{OBSERVER-BASED CONTROL DESIGN}

This section deals with the local exponential stabilization of system (11), (12) and equivalently with the local exponential stabilization of system (4), (6) by the switching law (5). Assumptions A.1, A.2 and A.3 are used to prove that there exist a switching matrix $\Gamma$ and an observer gain $L$ such that the system is locally exponentially stable. The results are given in the following.

Theorem 1: Assume that A.1, A.2 and A.3 hold. Then there exist matrices $\Gamma$ (characterizing the switching hyperplanes) and $L$ (the observer gain) such that system (11), (12) (or equivalently the closed-loop system (4), (5), (6)) is locally exponentially stable in an ellipsoidal domain containing the origin.

Proof: Since system (4) is stabilizable, then there exist a static gain $K$, a scalar $\alpha_{K}>0$ and a symmetric positive definite matrix $P_{1}$ such that $A_{c l}=A+B K$ is Hurwitz and satisfies

$$
A_{c l}^{T} P_{1}+P_{1} A_{c l} \preceq-2 \alpha_{K} P_{1} .
$$

Likewise, since the system is observable, then there exist an observer gain $L$, a scalar $\alpha_{o}>0$ and a symmetric positive definite matrix $P_{2}$ such that $A_{o}=A+L C$ is Hurwitz and satisfies

$$
A_{o}^{T} P_{2}+P_{2} A_{o} \preceq-2 \alpha_{o} P_{2} .
$$

We want to prove that the system (11), (12) is locally exponentially stable in some domain $\mathcal{D}$ around the origin. Let us consider the quadratic Lyapunov function

$$
V(\xi)=\xi^{T} P \xi
$$

with the $2 n \times 2 n$ matrix

$$
P=\left[\begin{array}{cc}
P_{1} & 0 \\
0 & \lambda P_{2}
\end{array}\right]
$$

with a scaling term $\lambda>0$. We want to show then that taking the matrix $\Gamma$ defined in (5) as

$$
\Gamma=P_{1} B=\left[\begin{array}{ll}
I & 0
\end{array}\right] P \tilde{B}
$$

with $\tilde{B}$ defined in (12) and for some positive scalar $\alpha$ we have

$$
\sup _{y \in \mathcal{F}[\mathcal{X}](\xi)} \frac{\partial V}{\partial \xi} y \leq-2 \alpha V(\xi),
$$

in a domain $\mathcal{D} \subset \mathbb{R}^{2 n}$ to be determined.

For each $\hat{x} \in \mathbb{R}^{n}$ we define the set of minimizers in which the control (5) takes values. This corresponds to defining minimizers in which the control (12) takes values such that

$$
\hat{x}^{T} \Gamma v=\hat{x}^{T} P_{1} B v=\xi^{T}\left[\begin{array}{l}
I \\
0
\end{array}\right] P \tilde{B} v .
$$

We define for any $z \in \mathbb{R}^{2 n}$ the set of indexes $\mathcal{I}^{*}(z)$ such that

$$
\mathcal{I}^{*}(z)=\left\{i \in \mathcal{I}_{N}: z^{T} P \tilde{B}\left(v_{j}-v_{i}\right) \geq 0, \forall j \in \mathcal{I}_{N}\right\},
$$

with $\tilde{B}$ defined in (11). To $\mathcal{I}^{*}(z)$ we associate for all $z \in \mathbb{R}^{2 n}$ the set $\Delta^{*}(z)$ of vectors defined by:

$$
\Delta^{*}(z)=\left\{\beta \in \Delta_{N}: \beta_{i}=0, \forall i \in \mathcal{I}_{N} \backslash \mathcal{I}^{*}(z)\right\} .
$$

Using (25) and (26), the set valued map $\mathcal{F}[\mathcal{X}](\xi)$ in (13) satisfies

$$
\mathcal{F}[\mathcal{X}](\xi) \subseteq \mathcal{F}^{*}[\mathcal{X}](\xi)
$$

with

$$
\begin{aligned}
\mathcal{F}^{*}[\mathcal{X}](\xi) & =\overline{\operatorname{Conv}_{i \in \mathcal{I}^{*}(\xi)}}\left\{\tilde{A} \xi+\tilde{B} v_{i}\right\} \\
& =\left\{\tilde{A} \xi+\tilde{B} v(\beta): \beta \in \Delta^{*}(\xi)\right\},
\end{aligned}
$$

with $v(\beta)=\sum_{i=1}^{N} \beta_{i} v_{i}$.

Consider the gain $K$ satisfying (18). From (27) and (28) and using the fact that $\Delta^{*}(\xi)$ is compact, we have

$$
\begin{aligned}
\sup _{y \in \mathcal{F}[\mathcal{X}](\xi)} \frac{\partial V}{\partial \xi} y & \leq \sup _{y \in \mathcal{F}^{*}[\mathcal{X}](\xi)} \frac{\partial V}{\partial \xi} y \\
& =\sup _{\beta \in \Delta^{*}(\xi)}\left\{\frac{\partial V}{\partial \xi}\{\tilde{A} \xi+\tilde{B} v(\beta)\}\right\} \\
& =\max _{\beta \in \Delta^{*}(\xi)}\left\{\frac{\partial V}{\partial \xi}\{\tilde{A} \xi+\tilde{B} v(\beta)\}\right\} .
\end{aligned}
$$

Thus, in order to show (23), it is sufficient to prove that for some scalar $\alpha>0$ we have

$$
\max _{\beta \in \Delta^{*}(\xi)}\left\{\frac{\partial V}{\partial \xi}\{\tilde{A} \xi+\tilde{B} v(\beta)\}\right\} \leq-2 \alpha V(\xi),
$$

in a domain $\mathcal{D}$ to be determined.

Note that, since Assumption A-2 holds, then there exists a neighborhood of the origin $\mathcal{E}(P, \gamma) \subset \mathbb{R}^{2 n}$, with $\gamma>0$ such that for all $\xi=\left[\begin{array}{l}\hat{x} \\ e\end{array}\right] \in \mathcal{E}(P, \gamma)$, we have

$$
K \hat{x}=\mathcal{K} \xi \in \operatorname{Conv}\{\mathcal{V}\},
$$

with $\mathcal{K}=\left[\begin{array}{ll}K & 0\end{array}\right]$.

Therefore, for all $\xi \in \mathcal{E}(P, \gamma)$ there exist scalars $\alpha_{j}(\xi)$, $j \in \mathcal{I}_{N}$ such that $\sum_{j=1}^{N} \alpha_{j}(\xi)=1$ and

$$
\mathcal{K} \xi=\sum_{j=1}^{N} \alpha_{j}(\xi) v_{j} .
$$


From (25), for all $i \in \mathcal{I}^{*}(\xi)$ we have

$$
\xi^{T} P \tilde{B}\left(v_{j}-v_{i}\right) \geq 0, \forall j \in \mathcal{I}_{N} .
$$

Then, for any $\beta \in \Delta^{*}(\xi)$, we have

$$
\xi^{T} P \tilde{B}\left(v_{j}-v(\beta)\right) \geq 0, \forall j \in \mathcal{I}_{N} .
$$

Then, considering (32), and multiplying the last inequalities by $\alpha_{j}(\xi)$ and summing the $N$ elements we obtain

$$
\xi^{T} P \tilde{B}(\mathcal{K} \xi-v(\beta)) \geq 0 .
$$

Adding this to the left part of (30), it comes

$$
\begin{aligned}
& \max _{\beta \in \Delta^{*}(\xi)}\left\{\frac{\partial V}{\partial \xi}\{\tilde{A} \xi+\tilde{B} v(\beta)\}\right\} \\
& \leq \max _{\beta \in \Delta^{*}(\xi)}\left\{2 \xi^{T} P\{\tilde{A} \xi+\tilde{B} v(\beta)\}\right\}+2 \xi^{T} P \tilde{B}(\mathcal{K} \xi-v(\beta)) \\
& =2 \frac{\partial V}{\partial \xi}\left[\begin{array}{cc}
A_{c l} & L C \\
0 & A_{o}
\end{array}\right] \xi=2 \frac{\partial V}{\partial \xi}\left(\tilde{A}_{c l} \xi\right)=2 \xi^{T} P\left(\tilde{A}_{c l} \xi\right) .
\end{aligned}
$$

Thus, in order to show (23), it is sufficient to prove that

$$
2 \xi^{T} P\left(\tilde{A}_{c l} \xi\right) \leq-2 \alpha V(\xi), \forall \xi \in \mathcal{E}(P, \gamma)
$$

which holds if

$$
\tilde{A}_{c l}^{T} P+P \tilde{A}_{c l} \preceq-2 \alpha P \text {. }
$$

Note that

$$
\begin{aligned}
& \tilde{A}_{c l}^{T} P+P \tilde{A}_{c l}+2 \alpha P= \\
& {\left[\begin{array}{cc}
A_{c l}^{T} P_{1}+P_{1} A_{c l}+2 \alpha P_{1} & P_{1} L C \\
(L C)^{T} P_{1} & \lambda\left(A_{o}^{T} P_{2}+P_{2} A_{o}+2 \alpha P_{2}\right)
\end{array}\right] .}
\end{aligned}
$$

Applying the Schur complement, the matrix (39) is negative if and only if

$$
A_{o}^{T} P_{2}+P_{2} A_{o}+2 \alpha P_{2} \preceq 0
$$

and

$$
\begin{aligned}
& \left(A_{c l}^{T} P_{1}+P_{1} A_{c l}+2 \alpha P_{1}\right) \\
& -\frac{1}{\lambda} P_{1} L C\left[2 \alpha P_{2}+A_{o}^{T} P_{2}+P_{2} A_{o}\right]^{-1}(L C)^{T} P_{1} \\
& \preceq 0 .
\end{aligned}
$$

Since (18) and (19) are satisfied, it is obvious that if we take $\alpha \leq \min \left(\alpha_{K}, \alpha_{o}\right)$, and $\lambda$ large enough both inequalities are verified.

Thus, there exist $\Gamma=P_{1} B$ and an observer gain $L$ such that system (11), (12) (and equivalently (4), (5), (6)) is locally $\alpha$-stable with a domain of attraction $\mathcal{E}(P, \gamma)$.

Remark 1: Note that, the proof of Theorem 1 is constructive in the sense that if the inequalities (18) and (19) are satisfied then the closed loop system (11), (12) is locally exponentially stable with an observer gain satisfying (19) and a switching hyperplane given by $\Gamma=P_{1} B$ with $P_{1}$ satisfying (18). Inequalities (18) and (19) can be easily converted to classical LMI design conditions [4]: there exist $Q_{1} \succ 0$, $P_{2} \succ 0, \mu>0, \theta>0$ such that

$$
\begin{gathered}
Q_{1} A^{T}+A Q_{1}-\theta B B^{T} \preceq-2 \alpha Q_{1}, \\
A^{T} P_{2}+P_{2} A-\mu C^{T} C \preceq-2 \alpha P_{2},
\end{gathered}
$$

with $Q_{1}=P_{1}^{-1}$. Then the matrix defining the switching hyperplanes is given by $\Gamma=Q_{1}^{-1} B$, and the observer gain is given by $L=-\frac{\mu}{2} C P_{2}^{-1}$.

Remark 2: Some results in the literature extend the classical separation principle to the case of linear system stabilized via an observer-based nonlinear controller [26]. However, only the case of continuous nonlinear controllers is considered. This property is not verified in the case of relay feedback controller. Note that conditions (42), (43) are independent i.e. they do not have cross variables. Furthermore, from (41) one may notice that when (42) and (43) are satisfied there always exist $\lambda$ and $\alpha$ such that (38) holds, that is the closed-loop system (18), (19) is stable. This means that the problems of observer synthesis and design of switching surfaces can be addressed independently. It represents a useful extension of the separation principle for systems with relays.

\section{NUMERICAL EXAMPLE}

Consider the linear system (4) with

$$
u \in \mathcal{V}=\{-v, v\}=\{-5,5\}
$$

and matrices

$$
A=\left[\begin{array}{cc}
-1.6 & 1.7 \\
1.5 & 2
\end{array}\right], B=\left[\begin{array}{l}
0 \\
1
\end{array}\right], \text { and } C=[1,0]
$$

The eigenvalues of $A$ are -2.2 , and 2.6 thus the open-loop linear system is unstable. Considering a decay rate $\alpha=5.5$ an observer based relay feedback controller is designed to stabilize the system to the origin.

After the implementation of the set of LMIs (42)-(43), we find that they are feasible for

$$
\begin{aligned}
& \theta=486.8634, Q_{1}=\left[\begin{array}{cc}
1.4334 & -4.7050 \\
-4.7050 & 28.6001
\end{array}\right], \\
& \mu=348.4742, P_{2}=\left[\begin{array}{cc}
37.3918 & -5.4278 \\
-5.4278 & 1.0098
\end{array}\right]
\end{aligned}
$$

Then, we compute the observer gain

$$
L=\left[\begin{array}{c}
-21.2 \\
-113.98
\end{array}\right]
$$

and the matrix characterizing the switching hyperplanes

$$
\Gamma=\left[\begin{array}{c}
0.25 \\
0.076
\end{array}\right]
$$

The computer simulations are performed for the initial conditions $x(0)=\left[\begin{array}{ll}1, & 0.5\end{array}\right]^{T}$, and $\hat{x}(0)=\left[\begin{array}{ll}0 & 0\end{array}\right]^{T}\left(\xi^{T}=\right.$ $\left.\left[\begin{array}{llll}0 & 0 & -1 & -0.5\end{array}\right]^{T}\right)$ and the results are reported in Figures $1-5$. 


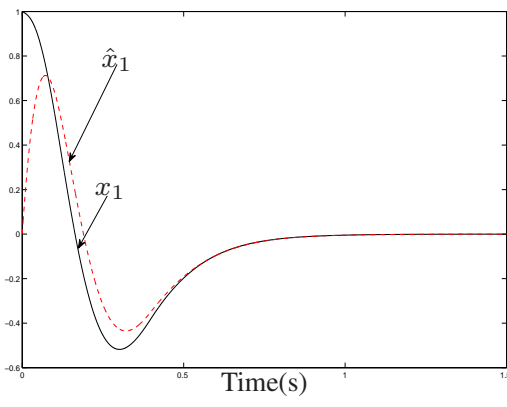

Fig. 1. Real state $x_{1}$ and its estimate $\hat{x}_{1}$

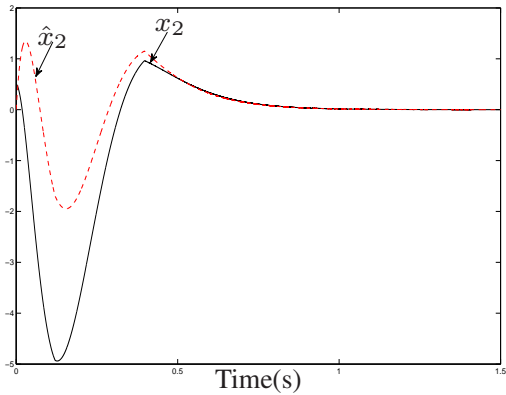

Fig. 2. Real state $x_{2}$ and its estimate $\hat{x}_{2}$

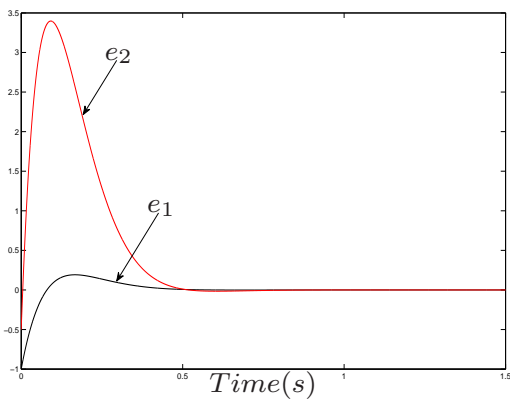

Fig. 3. Observation errors $e=\hat{x}-x$

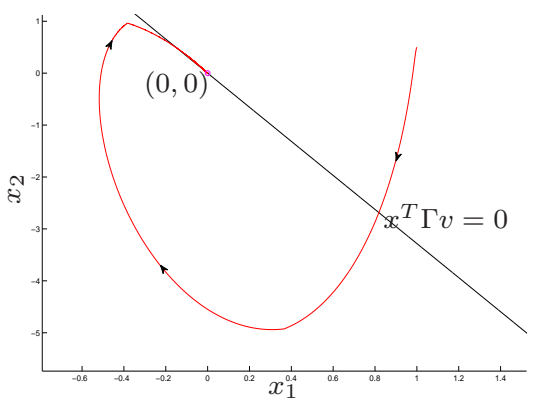

Fig. 4. $x_{1}$ and $x_{2}$ in the phase plot

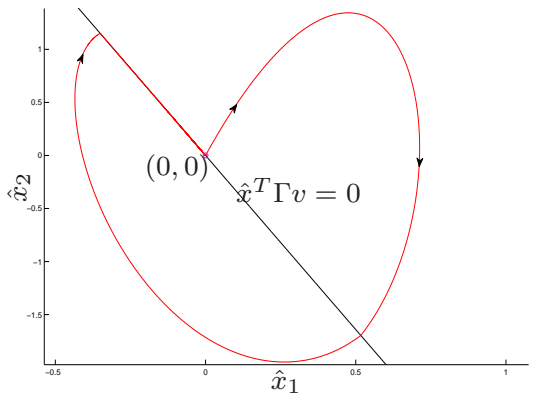

Fig. 5. $\hat{x}_{1}$ and $\hat{x}_{2}$ in the phase plot

As we can see from Figures 1 and 2, the states are exactly estimated and they converge to the origin and remain therein. From Figure 3, we can remark that the observation errors converge to zero exponentially, and then the estimated states converge to the real states. In Figure 5 the observer's phase portrait is presented together with the switching hyperplane $\hat{x}^{T} \Gamma v=0$. We can observe that the trajectory initialized at zero evolves until reaching the switching hyperplane and it slides over it. The hyperplane $x^{T} \Gamma v=0$ and the phase plot of the closed loop system (11), (12) are presented in Figure 4. Comparing Figure 4 and Figure 5, we can see that the hyperplane $x^{T} \Gamma v=0$ doesn't coincide exactly with the hyperplane $\hat{x}^{T} \Gamma v=0$. This is due to the fact that $\hat{x}$ converges to $x$ when $t$ tends to infinity. In simulations, the trajectory of the closed loop system reaches first the hyperplane $\hat{x}^{T} \Gamma v=0$ which tends to $x^{T} \Gamma v=0$ as $t$ goes to infinity and slides over it until reaching the origin. Note that, the quantitative estimation of the domain of attraction is not given in this paper. The problem of estimation and the optimization of the domain of attraction will be considered in future works.

\section{Conclusion}

In this article, the problem of designing an observer-based relay feedback controller for LTI systems is addressed. The control takes values from a finite set of constant vectors and the switching law depends on the estimated states. Qualitative conditions for the stability of the coupled plant are given. The assessment of the performance of the proposed observer-based relay feedback control algorithm is based on the simulation results. The problems of the estimation of the domain of attraction and the study of robustness with respect to perturbations will be studied in depth in future works.

\section{REFERENCES}

[1] J. P. Aubin and A. Cellina, Differential inclusions: set-valued maps and viability theory. Springer-Verlag New York, Inc., 1984.

[2] A. Bacciotti and L. Rosier, Liapunov functions and stability in control theory. Springer Science \& Business Media, 2006.

[3] I. Boiko, Discontinuous control systems: frequency-domain analysis and design. Springer Science \& Business Media, 2008.

[4] S. P. Boyd, L. El Ghaoui, E. Feron, and V. Balakrishnan, Linear matrix inequalities in system and control theory. SIAM, 1994, vol. 15.

[5] J. Cortes, "Discontinuous dynamical systems," Control Systems, vol. 28, no. 3, pp. 36-73, 2008. 
[6] R. Delpoux, L. Hetel, and A. Kruszewski, "Parameter-dependent relay control: Application to pmsm," Control Systems Technology, IEEE Transactions on, vol. 23, no. 4, pp. 1628-1637, July 2015.

[7] C. Edwards and S. Spurgeon, Sliding mode control: theory and applications. CRC Press, 1998.

[8] F.-L. EIrmgard, Discontinuous automatic control. Princeton NJ, 1953.

[9] A. F. Filippov and F. M. Arscott, Differential equations with discontinuous righthand sides: control systems. Springer Science \& Business Media, 1988, vol. 18.

[10] T. Floquet, C. Edwards, and S. K. Spurgeon, "An output feedback sliding mode control strategy for mimo systems of arbitrary relative degree," International Journal of Robust and Nonlinear Control, vol. 21, no. 2, pp. 119-236, 2011. [Online]. Available: https://hal.inria.fr/hal-00643773

[11] J. M. Gonçalves, A. Megretski, M. Dahleh et al., "Global stability of relay feedback systems," Transactions on Automatic Control, vol. 46, no. 4 , pp. $550-562,2001$

[12] S. Govindaswamy, L. Hetel, A. Polyakov, and T. Floquet, "On relay control for discrete time systems using linear matrix inequalities," in European Control Conference (ECC). IEEE, 2014, pp. 2225-2230.

[13] C. Hang, K. Astrom, and Q. Wang, "Relay feedback auto-tuning of process controllers: a tutorial review," Journal of Process Control, vol. 12 , no. 1 , pp. 143-162, 2002.

[14] L. Hetel, E. Fridman, and T. Floquet, "Variable structure control with generalized relays: A simple convex optimization approach," Transactions on Automatic Control, vol. 60, no. 2, pp. 497-502, 2015.

[15] K. H. Johansson, A. E. Barabanov, and K. J. Åström, "Limit cycles with chattering in relay feedback systems," Transactions on Automatic Control, vol. 47, no. 9, pp. 1414-1423, 2002.

[16] K. H. Johansson, A. Rantzer et al., "Fast switches in relay feedback systems," Automatica, vol. 35, no. 4, pp. 539-552, 1999.

[17] D. Liberzon, Switching in systems and control. Springer Science \& Business Media, 2003.

[18] T. Liu and F. Gao, Industrial process identification and control design: step-test and relay-experiment-based methods. Springer Science \& Business Media, 2011

[19] D. G. Luenberger, "Observers for multivariable systems," IEEE Transactions on Automatic Control, vol. 11, no. 2, pp. 190-197, 1966.

[20] D. Luenberger, "Observing the state of a linear system," IEEE Transactions on Military Electronics, vol. 8, no. 2, pp. 74-80, April 1964.

[21] W. Perruquetti and J.-P. Barbot, Sliding mode control in engineering. CRC Press, 2002.

[22] A. Polyakov, "Practical stabilization via relay delayed control," in Conference on Decision and Control. IEEE, 2008, pp. 5306-5311.

[23] — " "On practical stabilization of systems with delayed relay control," Automation and Remote Control, vol. 71, no. 11, pp. 2331-2344, 2010.

[24] A. Poznyak, A. Polyakov, and V. Azhmyakov, Attractive Ellipsoids in Robust Control. Springer, 2014.

[25] P. Riedinger, M. Sigalotti, and J. Daafouz, "Observabilité d'un convertisseur multi-niveaux via le principe d'invariance de lasalle," in Sixième Conférence Internationale Francophone d'Automatique (CIFA), 2010, p. CDROM.

[26] B. Ross Barmish and A. R. Galimidi, "Robustness of luenberger observers: Linear systems stabilized via non-linear control," Automatica, vol. 22, no. 4, pp. 413-423, 1986.

[27] I. Z. Tsypkin, Relay control systems. CUP Archive, 1984.

[28] V. Utkin, J. Guldner, and J. Shi, Sliding mode control in electromechanical systems. CRC press, 2009, vol. 34.

[29] Q.-G. Wang, T. H. Lee, and L. Chong, Relay feedback: analysis, identification and control. Springer Science \& Business Media, 2012.

[30] C.-C. Yu, Autotuning of PID controllers: A relay feedback approach. Springer Science \& Business Media, 2006. 